\title{
Value of an aggregate index in describing the impact of trends in antimicrobial resistance for Escherichia coli
}

\author{
David M Patrick MD FRCPC MHSc ${ }^{1,2}$, Catharine Chambers MSc ${ }^{1}$, Dale Purych $\mathrm{MD}^{3,4}$, Mei Chong MSc ${ }^{1}$, \\ Diana George $\mathrm{MSc}^{1}$, Fawziah Marra BSc PharmD FCSHP 5
}

DM Patrick, C Chambers, D Purych, M Chong, D George, F Marra. Value of an aggregate index in describing the impact of trends in antimicrobial resistance for Escherichia coli. Can J Infect Dis Med Microbiol 2015;26(1):33-38.

BACKGROUND: Drug resistance indexes (DRIs) quantify the cumulative impact of antimicrobial resistance on the likelihood that a given pathogen will be susceptible to antimicrobial therapy.

OBJECTIVE: To derive a DRI for community urinary tract infections caused by Escherichia coli in British Columbia for the years 2007 to 2010, and to examine trends over time and across patient characteristics.

METHODS: Indication-specific utilization data were obtained from BC PharmaNet for outpatient antimicrobial prescriptions linked to diagnostic information from physician payment files. Resistance data for $E$ coli urinary isolates were obtained from BC Biomedical Laboratories (now part of LifeLabs Medical Laboratory Services). DRIs were derived by multiplying the rate of resistance to a specific antimicrobial by the proportional rate of utilization for that drug class and aggregating across drug classes. Higher index values indicate more resistance.

RESULTS: Adaptive-use DRIs remained stable over time at approximately $18 \%$ (95\% CI $17 \%$ to $18 \%$ ) among adults $\geq 15$ years of age and approximately $28 \%$ (95\% CI $26 \%$ to $31 \%$ ) among children $<15$ years of age. Similar results were observed when proportional drug use was restricted to the baseline year (ie, a static-use model). Trends according to age group suggest a U-shaped distribution, with the highest DRIs occurring among children $<10$ years of age and adults $\geq 65$ years of age. Males had consistently higher DRIs than females for all age groups.

CONCLUSIONS: The stable trend in adaptive-use DRIs over time suggests that clinicians are adapting their prescribing practices for urinary tract infections to local resistance patterns. Results according to age group reveal a higher probability of resistance to initial therapy among young children and elderly individuals.

Key Words: Antimicrobial resistance; Drug resistance index; Escherichia coli

U rinary tract infections (UTIs) are common bacterial infections that affect both pediatric and adult patients in community and hospital settings. Escherichia coli is the most predominant uropathogen, causing approximately $80 \%$ of uncomplicated cystitis in adult females (1-3). Treatment of acute uncomplicated cystitis typically involves oral therapy with nitrofurantoin, with alternative therapies including cefixime, trimethoprim-sulfamethoxazole (TMP-SMX), trimethoprim or ciprofloxacin (4). However, the increasing proportion

\section{La valeur d'un indice composite pour décrire les effets des tendances de résistance antimicrobienne à l'Escherichia coli}

HISTORIQUE : Les indices de pharmacorésistance (IPR) quantifient l'effet cumulatif de la résistance antimicrobienne sur la probabilité qu'un pathogène donné soit susceptible à un traitement antimicrobien. OBJECTIF : Dériver l'IPR des infections urinaires d'origine non nosocomiale causées par l'Escherichia coli en Colombie-Britannique entre 2007 et 2010 et examiner les tendances au fil du temps et selon les caractéristiques des patients.

MÉTHODOLOGIE : Les données sur les indications d'utilisation, tirées du système PharmaNet de la Colombie-Britannique relativement aux prescriptions d'antimicrobiens, étaient liées à l'information diagnostique prélevée dans les dossiers d'honoraires des médecins. Les données de résistance reliées aux isolats urinaires d'E coli provenaient des BC Biomedical Laboratories (qui font désormais partie des LifeLabs Medical Laboratory Services). Les IPR étaient dérivés en multipliant le taux de résistance à un antimicrobien précis au taux proportionnel d'utilisation de cette classe de médicament et en les regroupant entre les classes de médicaments. Des indices de valeur plus élevés indiquaient une plus forte résistance.

RÉSULTATS : Les IPR à utilisation adaptée demeuraient stables au fil du temps, à environ $18 \%$ (95\% IC $17 \%$ à $18 \%$ ) chez les adultes de 15 ans et plus, et à environ $28 \%$ (95\% IC $26 \%$ à $31 \%)$ chez les enfants de moins de 15 ans. Les chercheurs ont observé des résultats similaires lorsque l'utilisation proportionnelle des médicaments était restreinte à l'année de référence (modèle à utilisation statique). Les tendances en fonction des groupes d'âge laissent supposer une répartition en U, les IPR les plus élevés se produisant chez les enfants de moins de dix ans et les adultes de 65 ans et plus. Dans tous les groupes d'âge, les hommes présentaient un IPR plus élevé que les femmes.

CONCLUSIONS : D'après la tendance stable des IPR à utilisation adaptée au fil du temps, les cliniciens adaptent leurs pratiques de prescription pour le traitement des infections urinaires aux profils de résistance locaux. Les résultats en fonction des groupes d'âge révèlent une plus forte probabilité de résistance à la thérapie initiale chez les jeunes enfants et les personnes âgées.

of uropathogens that are resistant to recommended antibiotic treatments challenges the appropriate care and management of UTIs and limits the potential benefits of these drugs $(5,6)$.

Evidence from national surveillance programs in Canada suggests that antimicrobial resistance among uropathogens has increased in recent years (7-9). In British Columbia (BC), there has been a ninefold increase in the proportion of E coli isolates nonsusceptible to ciprofloxacin from 1999 to 2011, with the highest resistance rates

\footnotetext{
${ }^{1}$ British Columbia Centre for Disease Control; ${ }^{2}$ School of Population and Public Health, University of British Columbia, Vancouver; ${ }^{3}$ LifeLabs

Medical Laboratory Services; ${ }^{4}$ Fraser Health Authority, Surrey; ${ }^{5}$ Faculty of Pharmaceutical Services, University of British Columbia,

Vancouver, British Columbia

Correspondence: Dr David M Patrick, BC Centre for Disease Control, 655 West 12th Avenue, Vancouver, British Columbia V5Z 4R4.

Telephone 604-707-2518, fax 604-707-2516, e-mail david.patrick@bccdc.ca

OPEN $\bigcirc$ ACCESS This open-access article is distributed under the terms of the Creative Commons Attribution Non-Commercial License (CC BY-NC) (http://
creativecommons.org/licenses/by-nc/4.0/), which permits reuse, distribution and reproduction of the article, provided that the original work is properly cited and the reuse is restricted to noncommercial purposes. For commercial reuse, contact support@pulsus.com
} 
observed among elderly age groups (10). In 2011, more than onequarter $(27 \%)$ of E coli isolates in BC were resistant to ciprofloxacin, more than one-quarter (26\%) were resistant to TMP-SMX and almost one-half (46\%) were resistant to ampicillin (10). Risk factors for developing a drug-resistant UTI include having had a previous UTI, previous antimicrobial exposure, recent hospitalization, longterm care residence, male sex, older age and comorbidities such as diabetes (11-15).

Ongoing surveillance of antimicrobial resistance trends is necessary to assess the overall burden of antimicrobial resistance over time and to derive empirical treatment guidelines for bacterial infections. However, conveying this information in an understandable and cohesive manner to policymakers and health care practitioners is challenging, particularly when resistance rates are examined separately for each drug and when trends are inconsistent across drug classes (16). Further challenges arise when these trends are interpreted in the absence of information on the proportional use of antimicrobials used to treat a given infection, or the availability and relative effectiveness of alternative treatments (16). A drug resistance index (DRI) can overcome these challenges by aggregating resistance to multiple drug classes into a single composite measure for a given bacterial species or type of infection. The purpose of the present analysis was to calculate a DRI for community UTIs caused by E coli in BC for the years 2007 to 2010, and to examine DRI trends over time and across patient demographics.

\section{METHODS}

\section{Data sources}

Antimicrobial utilization data were obtained from the BC PharmaNet database of outpatient prescriptions for oral antimicrobials for systematic use for the years 2007 to 2010. The PharmaNet database includes records of all outpatient prescriptions dispensed from community pharmacies to BC residents. It excludes over-the-counter medications, medications administered to inpatients in acute care hospitals, medication samples dispensed at a physician's office, and medications administered for veterinary or agricultural use. Antimicrobial utilization data were classified according to the WHO's Anatomical Therapeutic Chemical classification system (17).

To derive indication-specific antimicrobial utilization rates, antimicrobial prescription records were linked to diagnostic codes from the BC Medical Services Plan (MSP) files. The MSP files contain data for all medically necessary services provided by fee-for-service practitioners to $\mathrm{BC}$ residents covered under the provincial insurance program. Prescription records were linked to MSP records based on matching patient and physician identifiers when the prescription dispensing date occurred within five days of the practitioner service date. In instances in which more than one MSP record was extracted, the record with the most recent service date was used. Indications were defined according to the International Classification of Diseases, Ninth Edition (ICD-9) (18). PharmaNet-MSP linked data were restricted to ICD-9 diagnostic codes for cystitis (595) and its subcodes (595.0, 595.1, etc).

Antimicrobial resistance data for E coli urinary isolates were obtained from BC Biomedical Laboratories Ltd (part of LifeLabs Medical Service Laboratories as of 2013), a community-based laboratory practice serving the Vancouver Coastal and Fraser Health Authorities in BC, for the years 2007 to 2010. Testing of isolates conformed to Clinical and Laboratory Standards Institute guidelines (19).

Annual population estimates used in the calculation of antimicrobial utilization rates were obtained from the BC Health Data Warehouse (20).

\section{Analysis}

Analyses were performed separately for adults $\geq 15$ years of age and children $<15$ years of age. In adults, antimicrobial utilization rates were calculated as the defined daily dose (DDD) per 1000 population per day, where DDD represents the average maintenance dose per day for a drug used in its main indication in adults (17). In children, antimicrobial utilization rates were calculated as the total number of prescriptions per 1000 population per day because antimicrobial dosing in pediatric patients varies according to weight.

An adaptive-use DRI for E coli was calculated according to methods adapted from Laxminarayan and Klugman (16) using the formula:

$$
\mathrm{R}=\sum_{\mathrm{k}} \mathrm{p}_{\mathrm{k}}^{\mathrm{t}} \mathrm{q}_{\mathrm{k}}^{\mathrm{t}}
$$

where $\mathrm{p}_{\mathrm{k}}^{\mathrm{t}}$ is the rate of resistance to drug class $\mathrm{k}$ at time $\mathrm{t}$ and $\mathrm{q}_{\mathrm{k}}^{\mathrm{t}}$ is the proportional rate of use for drug class $\mathrm{k}$ used to treat cystitis at time $\mathrm{t}$. Higher index values indicate more resistance.

For contrast, a 'static use' model was used to measure the hypothetical DRI trend had proportional antimicrobial use remained unchanged over time (16). Differences between static and observed adaptive DRIs help gauge the contributions of physician-adaptation to preserving the value of initial empirical therapy. The static DRI was calculated using the proportional rate of use for drug class $k$ fixed to the baseline year of the analysis. To ensure comparability of results with those of previous studies, a sensitivity analysis was also performed using overall prescription data for any indication rather than prescription data specifically for the treatment of cystitis.

Anatomical Therapeutic Chemical drug classes were restricted to drug classes relevant to the treatment of UTIs caused by E coli, where data were available. These included: penicillins with extended spectrum (J01CA), first-generation cephalosporins (J01DB), thirdgeneration cephalosporins (J01DD), fluoroquinolones (J01MA), nitrofuran derivatives (J01XE) and sulphonamide/trimethoprim combinations (J01EE). An isolate was considered to be resistant to a drug class if it was found to be nonsusceptible to at least one antimicrobial agent within that class (21). It should be noted that cefotaxime resistant cut-off ranges were modified in 2010, but that standards otherwise remained constant throughout the study period.

Stratified analyses were performed according to patient demographic characteristics (ie, age group and sex). Trends in DRIs were assessed using the nonparametric Spearman Rank test. Percentile CIs were calculated using nonparametric bootstrap methods with $\mathrm{m}=1000$ simulations performed on an approximately $10 \%$ sample (PharmaNet $=$ 47,782 observations; $\mathrm{BC}$ Biomedical $=13,166$ observations) drawn at random from the full datasets. Ethics approval for the present analysis was obtained from the Clinical Research Ethics Board at the University of British Columbia (Vancouver, British Columbia). All analyses were performed using SAS version 9.2 (SAS Institute, USA).

\section{RESULTS}

Among both patient populations, the highest resistance rates were observed for ampicillin (43.8\% for adults and $46.9 \%$ for children) in the baseline year, followed by cephalothin (25.8\% and 20.1\%), TMP. SMX (25.1\% and 26.2\%) and ciprofloxacin (22.7\% and $7.1 \%)$. Resistance rates for cefotaxime (11.8\% and 6.5\%) and nitrofuratoin (3.3\% and $1.3 \%)$ remained relatively low for both patient populations across all study years. For adults, resistance rates were generally stable over time despite a trend toward increasing resistance to fluoroquinolones $(\mathrm{P}>0.05)$ (Figure 1A). For children, resistance rates for ampicillin significantly decreased over time $(\mathrm{rho}=-1.00 ; \mathrm{P}<0.01)$, while resistance rates for ciprofloxacin significantly increased ( $r h o=1.00 ; \mathrm{P}<0.01$ ) (Figure 1B). Resistance rates for all other included drug classes were stable $(\mathrm{P}>0.05)$.

Among adults, fluoroquinolones comprised the largest proportional use at $37.9 \%$ in the baseline year, followed by nitrofuran derivatives $(32.1 \%)$ and sulphonamide/trimethoprim combinations $(21.4 \%)$ (Figure 2A). Proportional use of fluoroquinolones (rho $=-1.00$; $\mathrm{P}<0.01$ ) and sulphonamide/trimethoprim combinations ( $\mathrm{rho}=-1.00$; $\mathrm{P}<0.01$ ) significantly decreased during the study period, corresponding to a significant increase in the proportional use of nitrofuran derivatives ( $r h o=1.00 ; \mathrm{P}<0.01$ ). 


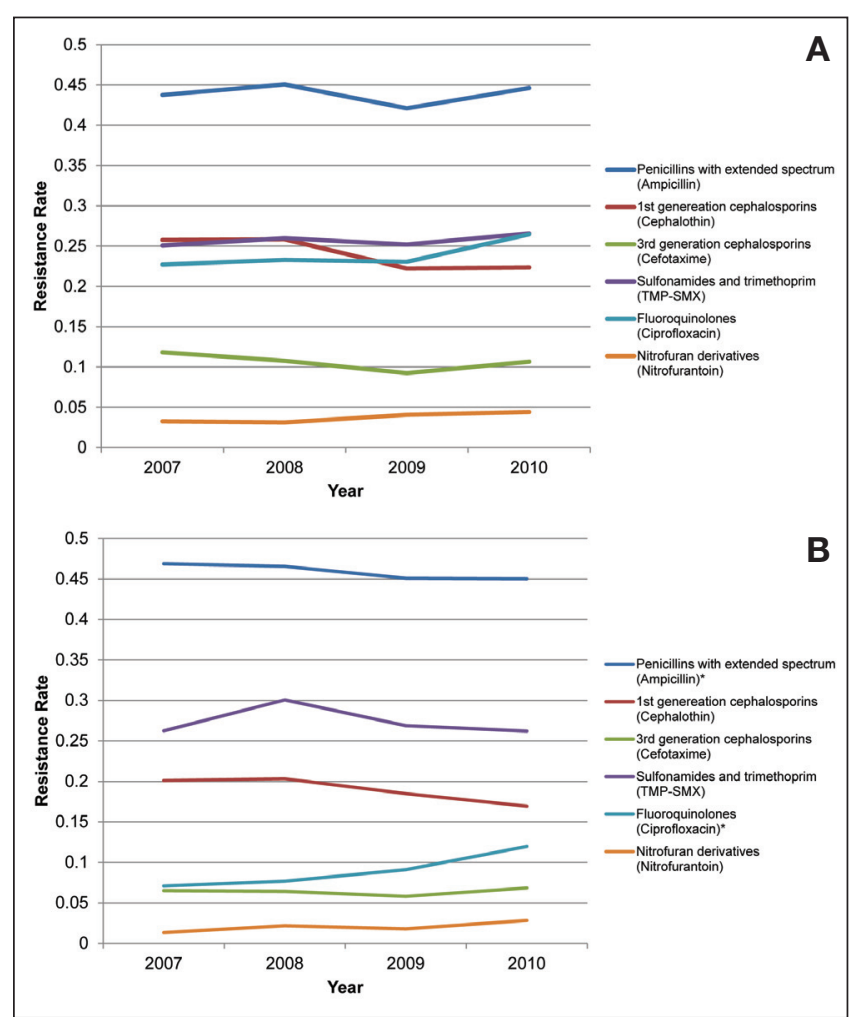

Figure 1) Proportion of Escherichia coli urinary isolates nonsusceptible to relevant drug classes in British Columbia among adults $\geq 15$ years of age (A) and children $<15$ years of age (B). *Trends over time are statistically significant according to nonparametric Spearman Rank test. TMP-SMX Trimethoprim-sulfamethoxazole

Among children, sulphonamide/trimethoprim combinations comprised the largest proportional drug use at $>50 \%$ across all study years (Figure 2B). A significant decreasing trend in proportional use was observed for sulphonamide/trimethoprim combinations over time (rho=-1.00; $\mathrm{P}<0.001)$, while significant increasing trends were observed for third-generation cephalosporins $(r h o=1.00 ; \mathrm{P}<0.001)$ and nitrofuran derivatives ( $\mathrm{rho}=1.00 ; \mathrm{P}<0.001)$.

Among adults, the adaptive-use DRI, when averaged across all study years, was 0.18 (95\% CI 0.17 to 0.18 ) and remained stable over time $(\mathrm{rho}=-0.80 ; \mathrm{P}=0.20)$ (Figure $3 \mathrm{~A})$. The static-use DRI increased from 0.18 ( $95 \%$ CI 0.17 to 0.19 ) in 2007 to 0.20 (95\% CI 0.19 to 0.21 ) in 2010; however, the overall trend was not significant $(\mathrm{rho}=0.80$; $\mathrm{P}=0.20$ ). DRI values for the static-use model did not significantly differ from the adaptive-use model over the four years of study, as indicated by the overlapping $95 \%$ CIs. DRI values did not dramatically differ when total number of prescriptions (versus DDDs) was used to calculate proportional antimicrobial use for adults (data not shown).

Among children, the adaptive-use DRI peaked at 0.30 (95\% CI 0.25 to 0.35$)$ in 2008 then declined to 0.27 (95\% CI 0.22 to 0.32 ) in 2010; however, this trend was not significant ( $\mathrm{rho}=-0.40 ; \mathrm{P}=0.60$ ) (Figure 3B). Nearly identical DRI values were observed for the staticuse model.

Fluoroquinolones and sulphonamide/trimethoprim combinations made the largest contribution to the DRI among adults across all years, while among children the trend in DRI values was predominantly driven by sulphonamide/trimethoprim combinations and penicillins with extended spectrum (Table 1). Other drug classes had relatively less impact on DRI trends among both adults and children. Analyses according to age group and sex show that DRI values were consistently higher among males than females across all age groups (Figure 4). For adults, there was a significant increasing trend from $0.09(95 \% \mathrm{CI}$ 0.08 to 0.11 ) among those 15 to 19 years of age to 0.32 (95\% CI

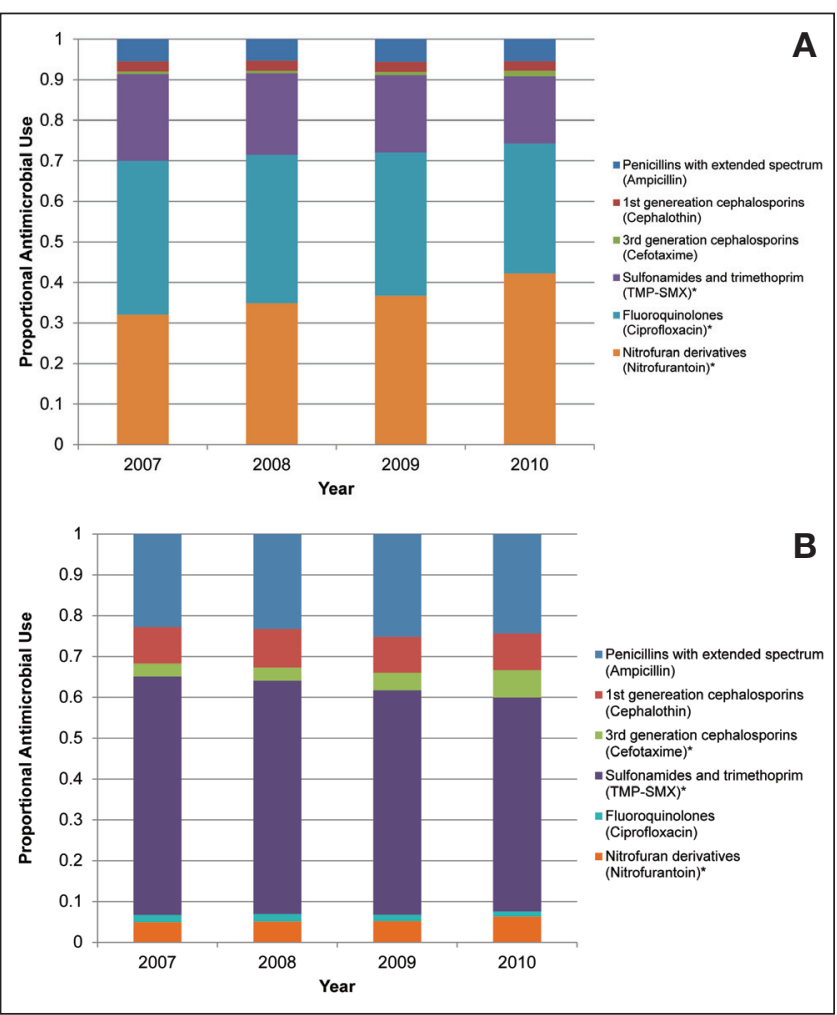

Figure 2) Proportional rate of antimicrobial use for cystitis in British Columbia among adults $\geq 15$ years of age (A) and children $<15$ years of age (B). *Trends over time are statistically significant according to nonparametric Spearman Rank test. TMP-SMX Trimethoprim-sulfamethoxazole

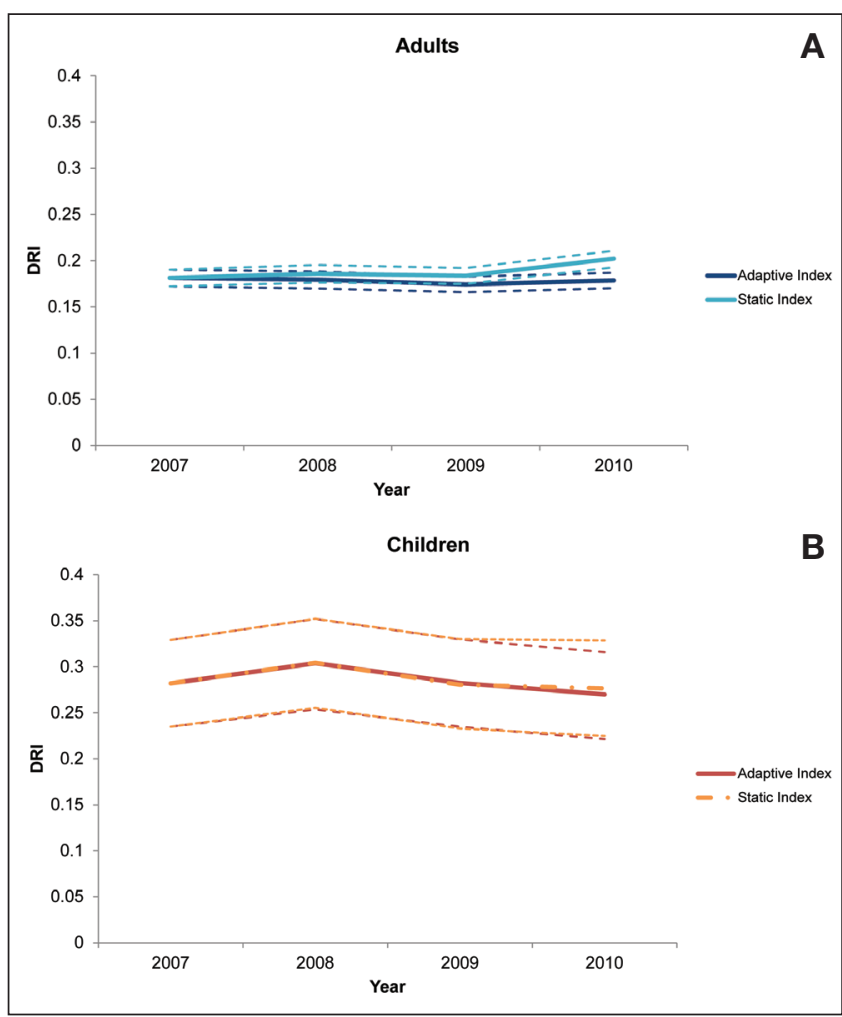

Figure 3) Adaptive- and static-use drug resistance indexes (DRIs) for Escherichia coli with 95\% CIs (shown as dashed lines) in British Columbia among adults $\geq 15$ years of age (A) and children $<15$ years of age (B) 
TABLE 1

Resistance rates for Escherichia coli urinary isolates and proportional rates of antimicrobial use for cystitis among adults $\geq 15$ years of age and children $<15$ years of age in British Columbia, 2007-2010

\begin{tabular}{|c|c|c|c|c|}
\hline Drug class (antibiotic) & Resistance rate (A), \% & $\begin{array}{c}\text { Proportional antimicrobial } \\
\text { use (B), \% }\end{array}$ & $\begin{array}{c}\text { Drug resistance index } \\
\left(A^{*} B\right)\end{array}$ & $\begin{array}{l}95 \% \mathrm{Cl} \text { for drug } \\
\text { resistance index }\end{array}$ \\
\hline \multicolumn{5}{|l|}{ Adults } \\
\hline Penicillins with extended spectrum (ampicillin) & 44.3 & 5.5 & 0.02 & \\
\hline First-generation cephalosporins (cephalothin) & 24.3 & 2.4 & 0.01 & \\
\hline Third-generation cephalosporins (cefotaxime) & 10.6 & 0.9 & 0.00 & \\
\hline Sulfonamides and trimethoprim (TMP-SMX) & 25.8 & 19.1 & 0.05 & \\
\hline Fluoroquinolones (ciprofloxacin) & 24.7 & 35.3 & 0.09 & \\
\hline Nitrofuran derivatives (nitrofurantoin) & 3.8 & 36.7 & 0.01 & \\
\hline Sum & & & 0.18 & $0.17-0.18$ \\
\hline \multicolumn{5}{|l|}{ Children } \\
\hline Penicillins with extended spectrum (ampicillin) & 45.8 & 23.9 & 0.11 & \\
\hline First-generation cephalosporins (cephalothin) & 19.3 & 9.1 & 0.02 & \\
\hline Third-generation cephalosporins (cefotaxime) & 6.2 & 4.4 & 0.00 & \\
\hline Sulfonamides and trimethoprim (TMP-SMX) & 27.1 & 55.6 & 0.15 & \\
\hline Fluoroquinolones (ciprofloxacin) & 9.2 & 1.6 & 0.00 & \\
\hline Nitrofuran derivatives (nitrofurantoin) & 2.0 & 5.4 & 0.00 & \\
\hline Sum & & & 0.28 & $0.26-0.31$ \\
\hline
\end{tabular}

TMP-SMX Trimethoprim-sulfamethoxazole

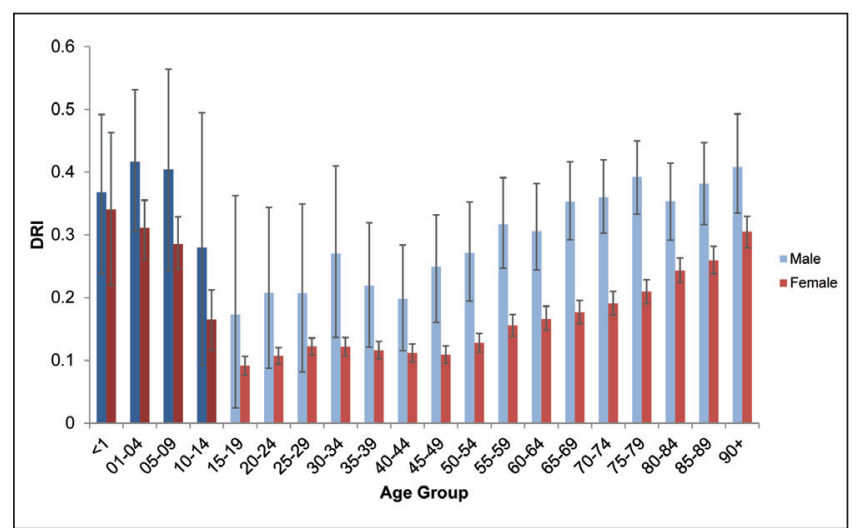

Figure 4) Adaptive-use drug resistance index (DRI) with 95\% CIs for Escherichia coli in British Columbia according to age group and sex

0.29 to 0.34$)$ among those $>90$ years of age $(\mathrm{rho}=0.95 ; \mathrm{P}<0.01)$. For children, there was a significant declining trend from 0.35 (95\% CI 0.27 to 0.43 ) among those $<1$ year of age to 0.17 (95\% CI 0.13 to 0.22 ) among those 10 to 14 years of age ( $r h o=-1.00 ; \mathrm{P}<0.01)$.

Sensitivity analysis

A sensitivity analysis was performed using overall, rather than indication-specific, prescription data to calculate proportional drug use. Among both patient populations, penicillins with extended spectrum were the most frequently used antimicrobial class for any indication in the baseline year ( $43.4 \%$ for adults and $75.0 \%$ for children), followed by fluoroquinolones $(22.1 \%$ and $0.4 \%$, respectively), firstgeneration cephalosporins $(14.8 \%$ and $12.4 \%$, respectively), sulphonamide/trimethprim combinations $(11.5 \%$ and $9.6 \%$, respectively), nitrofuran derivatives ( $7.5 \%$ and $0.4 \%$, respectively) and thirdgeneration cephalosporins $(0.7 \%$ and $2.2 \%$, respectively). Among adults, the adaptive-use DRI was significantly higher at 0.31 (95\% CI 0.31 to 0.32 ) when overall prescription data were used. The adaptiveuse DRI remained stable over time $(r h o=0.40 ; P=0.60)$ and did not significantly differ from the static-use model. Among children, the adaptive-use DRI was significantly higher at approximately 0.40 (95\% CI 0.37 to 0.43 ) when overall prescription data were used and remained stable over time $(\mathrm{rho}=-0.80 ; \mathrm{P}=0.20)$.

\section{DISCUSSION}

The DRI reflects the proportion of prescriptions for E coli cystitis in a population that uses a drug to which the organism is not susceptible. Our findings show that the overall DRI value for community E coli urinary isolates, when averaged across study years, is $18 \%$ (95\% CI $17 \%$ to $18 \%$ ) among adults $\geq 15$ years of age. This value is lower than the E coli DRI of 30\% (95\% CI 29\% to 32\%) reported by Laxminarayan and Klugman (16) for 2006, likely because our study used indicationspecific antimicrobial utilization data, which will more accurately reflect the tailored selection of antimicrobials for the treatment of UTIs. Sensitivity analyses based on overall prescribing for any indication found DRI values more consistent with previously reported values at $31 \%$ (95\% CI $31 \%$ to $32 \%$ ), largely driven by the greater proportional use of extended-spectrum penicillins. Our analyses were stratified according to age group, which may also partially explain the discrepancy with previous findings. While resistance rates were similar between adults $\geq 15$ years of age and children $<15$ years of age, the higher proportional use of extended-spectrum pencillins and sulphonamide/trimethoprim combinations among children increased the relative contribution of these drug classes to the age-specific DRI. For children, the overall DRI value across all study years was $28 \%$ (95\% CI 26\% to 31\%). Geographical variability may also be a factor because resistance rates are known to be significantly higher in the United States in comparison with Canada $(8,9)$.

Adaptive-use DRI trends remained stable over time, and DRI values based on a static-use model that fixes proportional antimicrobial use to the baseline year did not significantly differ from an adaptiveuse model. However, even over this short study period, the static index appears to be trending upward. A contrast between the value of the observed adaptive and static DRI is consistent with physicians increasing the proportionate use of nitrofurantoin. Physicians appear to have been increasing their use of this drug at the expense of TMP-SMX and ciprofloxacin. This change could be in response to evolving guidelines, emphasis in continuing education sessions or individual physician attention to antibiograms. Whatever the driver, physicians may be adapting prescribing practices to local resistance patterns (16). However, the limited availability of data makes it difficult to assess long-term trends. Others have noted rising resistance rates over time associated with the clonal spread of virulent, multidrug-resistant strains $(7,22)$, including extended-spectrum betalactamase-producing strains of community-acquired $E$ coli $(23,24)$. A 
longer duration of surveillance will be required to monitor the impact of extended-spectrum beta-lactamase-producing E coli strains on DRI trends in BC.

Consistent with recent guidelines $(4,25)$, our data suggest that nitrofurantoin is an appropriate treatment for acute uncomplicated cystitis given its low observed resistance rates. While this may be good news for cystitis, nitrofurantoin is not indicated for the treatment of pyelonephritis due to inadequate concentrations achieved in renal tissues and should not be used if early pyelonephritis is suspected $(4,25)$. Despite earlier research suggesting that nitrofurantoin has lower clinical cure rates and more side effects than traditional first-line agents, namely TMP-SMX and fluoroquinolones (26), more recent studies suggest that empirical treatment with nitrofurantoin is as effective as these drugs (25). Given the concerning resistance trends observed for ciprofloxacin and TMP-SMX, our findings support calls made by others for more judicious use of these drugs $(8,25-27)$. Routine testing data were not available for our study period for other, alternative treatments for UTIs, such as fosfomycin, which has only recently become available again in Canada.

DRI trends according to age group suggest a U-shaped distribution with the lowest rates occurring among adults $<50$ years of age and the highest rates occurring among young children and the elderly. Elderly adults are at greater risk for developing a drug-resistant infection, likely as a result of cumulative lifetime exposure to antimicrobials, particularly to fluoroquinolones, higher frequency and longer duration of hospital stays, increased likelihood of developing complicated infections and increased prevalence of comorbidities $(7,11,12,14)$. While acute, uncomplicated UTIs are generally self-limiting and not associated with long-term medical sequelae in adults in the absence of underlying conditions $(1,28)$, among pediatric patients, UTIs can be associated with significant morbidity, including impaired renal function and end-stage renal disease $(28,29)$.

Males had consistently higher DRI values than females across all age groups, a finding that is consistent with resistance trends observed nationally (7-9). These trends are largely driven by the greater proportional use of fluoroquinolones for cystitis and the higher rates of ciprofloxacin resistance among men (data not shown). The high prevalence of complicated UTIs among men, combined with the longer duration of therapies and older age at diagnosis, likely explains the higher observed resistance rates $(7,30,31)$. In children, this sex difference is likely the result of congenital abnormalities, which are more prevalent among males than females $(29,30)$.

The present study extends previous work in this area in a number of important ways. Access to prescription drug use through BC PharmaNet allows for examination of antimicrobial utilization in community settings for the entire population of BC. We have adopted standardized methods of measuring antimicrobial use in units of DDDs (17) and established linkage methodologies to connect antimicrobial prescriptions to ICD-9 diagnostic codes to derive indication-specific antimicrobial prescribing (32,33). However, despite these strengths, certain limitations should be acknowledged. Prescription data from PharmaNet are restricted to oral antimicrobials dispensed in outpatient settings. While these data comprehensively measure prescription use in the community - the main focus of our study - they do not capture antimicrobial use for agents that are administered parenterally

\section{REFERENCES}

1. Foxman B. The epidemiology of urinary tract infection. Nat Rev Urol 2010;7:653-60.

2. Nicolle LE. Uncomplicated urinary tract infection in adults including uncomplicated pyelonephritis. Urol Clin North Am 2008;35:1-12

3. Ronald A. The etiology of urinary tract infection: Traditional and emerging pathogens. Am J Med 2002;113(Suppl 1A):14S-9S.

4. Blondel-Hill E, Fryters S. Bugs and Drugs: An Antimicrobial/ Infectious Disease Reference, 2012 edn. Edmonton: Alberta Health Services, 2012.

5. Cohen ML. Epidemiology of drug resistance: Implications for a post-antimicrobial era. Science 1992;257:1050-5. and/or primarily used in inpatient settings. As a result, utilization rates for certain drug classes may be underestimated in our study or, in the case of carbapenems and aminoglycosides, excluded completely. The accuracy of data on indication-specific prescribing will depend on the manner in which physicians code the diagnosis when billing. We focused on the majority of UTIs for which a cystitis code (ICD-9 595) was coded to assure specificity of that diagnosis in describing treatment patterns, UTIs identified by less specific codes (eg, ICD-9 599) were excluded. Nonetheless, our linked data analysis reflects patterns of prescription for an entire population. BC Biomedical Laboratories are located in only two of five of the regional health authorities in $\mathrm{BC}$, and, although one of the largest laboratory service providers in $\mathrm{BC}$, they are not the sole provider of outpatient laboratory services in these areas. However, we have observed only limited variation in prescribing by region in $\mathrm{BC}$. Future analyses will begin with more complete sampling of laboratory data through collaboration with broader laboratory systems.

Routine susceptibility testing of penicillins with beta-lactamase inhibitors (eg, amoxicillin-clavulanate) was only performed from 2009 onwards; consequently, this antimicrobial class was excluded from our analysis to ensure comparability of DRI values over time. Culture samples are generally not indicated for young, otherwise healthy, premenopausal women. As such, isolates may be more representative of certain patient populations with higher resistance rates such as those with complicated UTIs (7-9). Finally, DRIs for adults and children in the present analysis may not be directly comparable due to differences in their calculations (refer to the Methods section).

\section{CONCLUSIONS}

DRIs improve our ability to quantify and communicate the cumulative impact of antimicrobial resistance on the likelihood that an organism will be susceptible to initial antimicrobial treatment. For clinicians, they provide information on the effectiveness of alternative treatment regimens and the relative adaptability of these treatments to local resistance patterns. For policymakers and other nonexpert groups, DRIs function as an important communication tool for translating knowledge about antimicrobial resistance into practice. Our findings show that the overall DRI value for community UTIs caused by E coli in BC is 18\% for adults and 28\% for children for the years 2007 to 2010. The likelihood that a UTI caused by E coli will be susceptible to initial antimicrobial therapy is highest among adult females, while concerning DRI values were observed among young children and the elderly. Further work is required to develop a DRI to summarize the impact of resistance on all relevant pathogens causing UTI in a population.

ACKNOWLEDGEMENTS: The authors thank collaborators who provided data required to conduct this study: the BC Ministry of Health and BC Biomedical Laboratories Ltd (part of LifeLabs Medical Service Laboratories Ltd as of 2013). The authors also acknowledge Ramanan Laxminarayan and Nikolay Braykov at the Center for Disease Dynamics, Economics and Policy in Washington, DC for their input on the methods.

INSTITUTION FROM WHICH WORK ORIGINATED: BC Centre for Disease Control.

6. Kunin CM. Resistance to antimicrobial drugs - a worldwide calamity. Ann Intern Med 1993;118:557-61.

7. Karlowsky JA, Lagace-Wiens PR, Simner PJ, et al. Antimicrobial resistance in urinary tract pathogens in Canada from 2007 to 2009: CANWARD surveillance study. Antimicrob Agents Chemother 2011;55:3169-75.

8. Zhanel GG, Hisanaga TL, Laing NM, et al. Antibiotic resistance in outpatient urinary isolates: Final results from the North American Urinary Tract Infection Collaborative Alliance (NAUTICA). Int J Antimicrob Agents 2005;26:380-8.

9. Zhanel GG, Hisanaga TL, Laing NM, et al. Antibiotic resistance in Escherichia coli outpatient urinary isolates: Final results from the 
North American Urinary Tract Infection Collaborative Alliance (NAUTICA). Int J Antimicrob Agents 2006;27:468-75.

10. British Columbia Centre for Disease Control. Antimicrobial resistance trends in the province of British Columbia, 2011. Vancouver: Communicable Disease Prevention and Control Services, British Columbia Centre for Disease Control, 2012.

11. Ben Ami R, Rodriguez-Bano J, Arslan H, et al. A multinational survey of risk factors for infection with extended-spectrum beta-lactamase-producing enterobacteriaceae in nonhospitalized patients. Clin Infect Dis 2009;49:682-90.

12. Colodner R, Rock W, Chazan B, et al. Risk factors for the development of extended-spectrum beta-lactamase-producing bacteria in nonhospitalized patients. Eur J Clin Microbiol Infect Dis 2004;23:163-7.

13. Colodner R, Kometiani I, Chazan B, Raz R. Risk factors for community-acquired urinary tract infection due to quinolone-resistant E. coli. Infection 2008;36:41-5.

14. Sotto A, De Boever CM, Fabbro-Peray P, Gouby A, Sirot D, Jourdan J. Risk factors for antibiotic-resistant Escherichia coli isolated from hospitalized patients with urinary tract infections: A prospective study. J Clin Microbiol 2001;39:438-44.

15. Wright SW, Wrenn KD, Haynes ML. Trimethoprim-sulfamethoxazole resistance among urinary coliform isolates. J Gen Intern Med 1999;14:606-9.

16. Laxminarayan R, Klugman KP. Communicating trends in resistance using a drug resistance index. BMJ Open 2011;1:e000135.

17. WHO Collaborating Centre for Drug Statistics Methodology. Guidelines for ATC classification and DDD assignment, 2012. Oslo: World Health Organization, 2011.

18. American Medical Association. International Classification of Diseases, 9th Revision, Clinical Modification. 1997.

19. Clinical and Laboratory Standards Institute. Performance standards for antimicrobial susceptibility testing: 19th information supplement. CLSI document M100-S19. Wayne: Clinical and Laboratory Standards Institute, 2009.

20. BC Stats. Population projections. <www.bcstats.gov.bc.ca/ StatisticsBySubject/Demography/PopulationProjections.aspx $>$ (Accessed January 9, 2013).

21. Magiorakos AP, Srinivasan A, Carey RB, et al. Multidrug-resistant, extensively drug-resistant and pandrug-resistant bacteria:

An international expert proposal for interim standard definitions for acquired resistance. Clin Microbiol Infect 2012;18:268-81.
22. Gupta K. Addressing antibiotic resistance. Am J Med 2002;113(Suppl 1A):29S-34S.

23. Nicolas-Chanoine MH, Blanco J, Leflon-Guibout V, et al. Intercontinental emergence of Escherichia coli clone O25:H4-ST131 producing CTX-M-15. J Antimicrob Chemother 2008;61:273-81.

24. Pitout JD, Nordmann P, Laupland KB, Poirel L. Emergence of Enterobacteriaceae producing extended-spectrum beta-lactamases (ESBLs) in the community. J Antimicrob Chemother 2005;56:52-9.

25. Gupta K, Hooton TM, Naber KG, et al. International clinical practice guidelines for the treatment of acute uncomplicated cystitis and pyelonephritis in women: A 2010 update by the Infectious Diseases Society of America and the European Society for Microbiology and Infectious Diseases. Clin Infect Dis 2011;52:e103-e120.

26. Hooton TM, Bradley SF, Cardenas DD, et al. Diagnosis, prevention, and treatment of catheter-associated urinary tract infection in adults: 2009 International Clinical Practice Guidelines from the Infectious Diseases Society of America. Clin Infect Dis 2010;50:625-63.

27. Naber KG, Schito G, Botto H, Palou J, Mazzei T. Surveillance study in Europe and Brazil on clinical aspects and Antimicrobial Resistance Epidemiology in Females with Cystitis (ARESC): Implications for empiric therapy. Eur Urol 2008;54:1164-75.

28. Stamm WE. Scientific and clinical challenges in the management of urinary tract infections. Am J Med 2002;113(Suppl 1A):1S-4S.

29. Ma JF, Shortliffe LM. Urinary tract infection in children: Etiology and epidemiology. Urol Clin North Am 2004;31:517-26.

30. Neal DE, Jr. Complicated urinary tract infections. Urol Clin North Am 2008;35:13-22.

31. Nicolle LE. Complicated urinary tract infection in adults. Can J Infect Dis Med Microbiol 2005;16:349-60.

32. Fuertes EI, Henry B, Marra F, Wong H, Patrick DM. Trends in antibiotic utilization in Vancouver associated with a community education program on antibiotic use. Can J Public Health 2010;101:304-8.

33. McKay RM, Vrbova L, Fuertes E, et al. Evaluation of the Do Bugs Need Drugs? program in British Columbia: Can we curb antibiotic prescribing? Can J Infect Dis Med Microbiol 2011;22:19-24. 


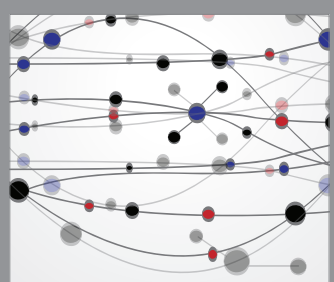

The Scientific World Journal
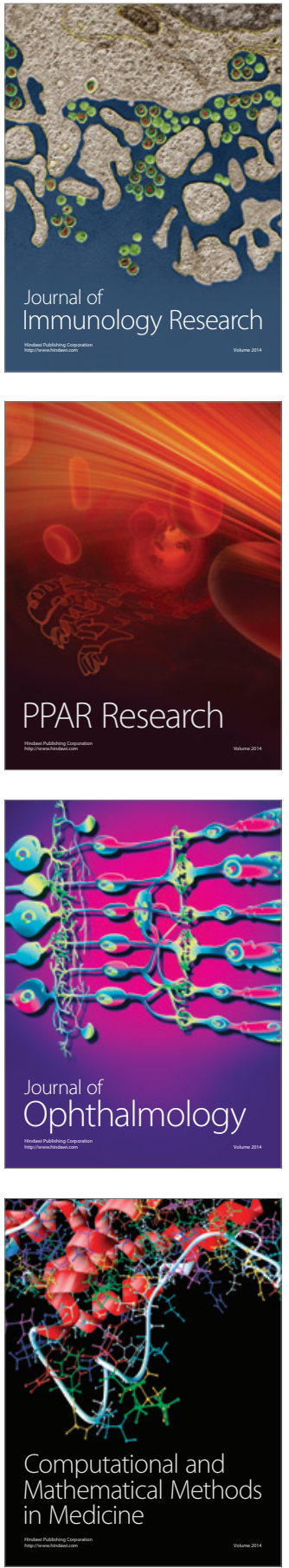

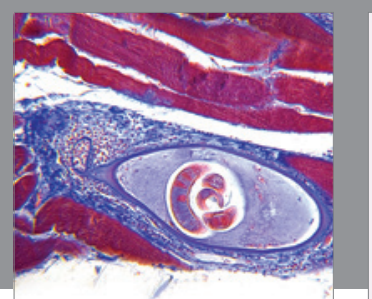

Gastroenterology Research and Practice

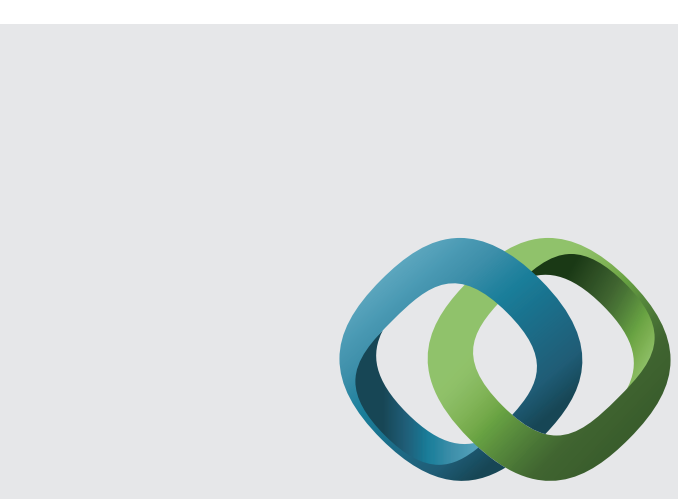

\section{Hindawi}

Submit your manuscripts at

http://www.hindawi.com
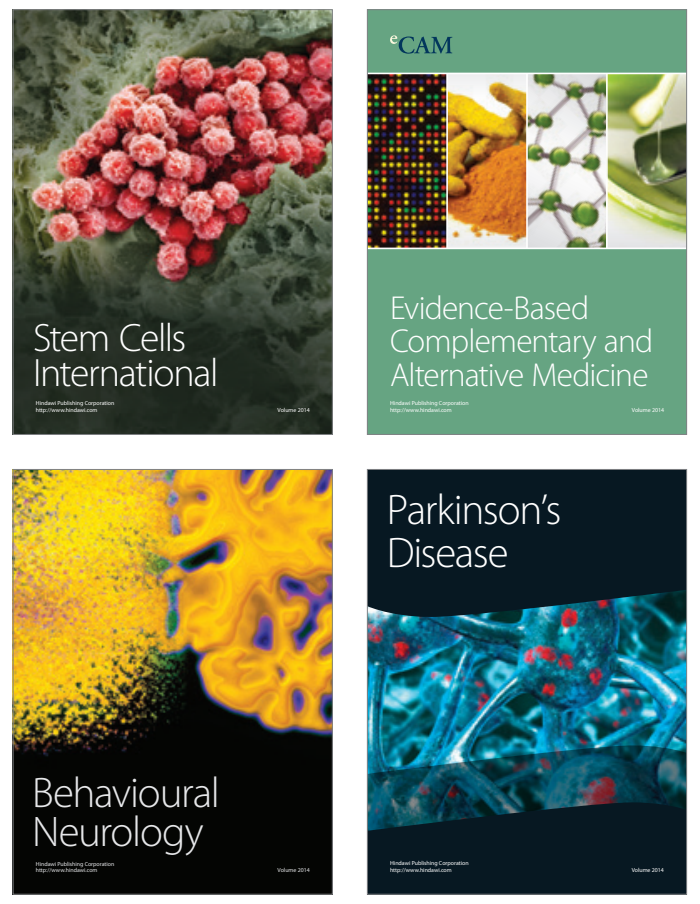
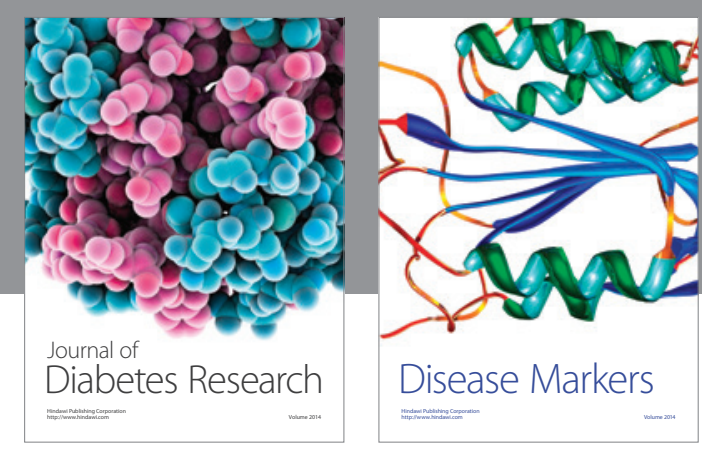

Disease Markers
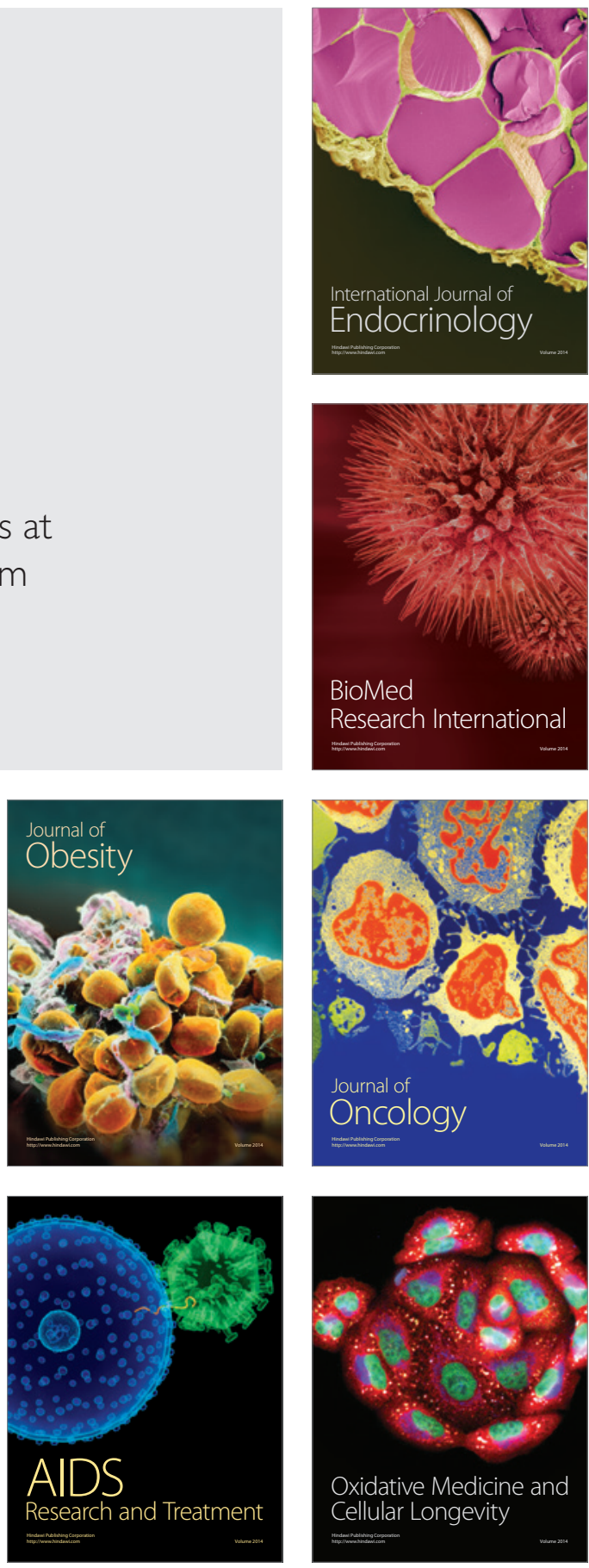